\title{
Symmetric 2-Structures, a Classification
}

\author{
Helmut Karzel, Jarosław Kosiorek and Andrzej Matraś \\ Dedicated to Momme Thomsen on the occasion of his 70th birthday
}

\begin{abstract}
We classify symmetric 2-structures $\left(P, \mathfrak{G}_{1}, \mathfrak{G}_{2}, \mathfrak{K}\right)$, i.e. chain structures which correspond to sharply 2-transitive permutation sets $(E, \Sigma)$ satisfying the condition: "(*) $\forall \sigma, \tau \in \Sigma: \sigma \circ \tau^{-1} \circ \sigma \in \Sigma$ ". To every chain $K \in \mathfrak{K}$ one can associate a reflection $\widetilde{K}$ in $K$. Then $(*)$ is equivalent to " $(* *) \forall K \in \mathfrak{K}: \widetilde{K}(\mathfrak{K})=\mathfrak{K}$ " and one can define an orthogonality " $\perp$ " for chains $K, L \in \mathfrak{K}$ by " $K \perp L \Leftrightarrow K \neq L \wedge \widetilde{K}(L)=L$ ". The classification is based on the cardinality of the set of chains which are orthogonal to a chain $K$ and passing through a point $p$ of $K$. For one of these classes (called point symmetric 2-structures) we proof that in each point there is a reflection and that the set of point reflections forms a regular involutory permutation set.
\end{abstract}

Mathematics Subject Classification (2000). 51B20(2000).

Keywords. Net, 2-structure, symmetric chain structure, midpoint.

\section{Introduction}

Let $S$ be a non empty set, $P:=S \times S$ the product set and let $\mathfrak{G}_{1}:=\{S \times\{x\} \mid$ $x \in S\}$ and $\left.\mathfrak{G}_{2}:=\{\{x\} \times S\} \mid x \in S\right\}$ be the sets of generators. Then $\left(P, \mathfrak{G}_{1}, \mathfrak{G}_{2}\right)$ is a net, i.e. $\forall p \in P, \forall i \in\{1,2\} \exists_{1} X \in \mathfrak{G}_{i}$ such that $p \in X$ (which we denote by $[p]_{i}:=X$ ) and if $X \in \mathfrak{G}_{1}, Y \in \mathfrak{G}_{2}$ then $X \cap Y \neq \emptyset$. A subset $C \subset P$ is called a chain if $\forall X \in \mathfrak{G}_{1} \cup \mathfrak{G}_{2}:|C \cap X|=1$. Let $\mathfrak{C}$ be the set of all chains of the net $\left(P, \mathfrak{G}_{1}, \mathfrak{G}_{2}\right)$, for $a, b \in P$ let $a \square b:=c$ with $\{c\}=[a]_{1} \cap[b]_{2}$ (we will also write $a b:=a \square b$ ). Let $P^{(2)}:=\left\{(a, b) \in P^{2} \mid a \square b \neq a, b\right\}$ and if $A, B, C \in \mathfrak{C}$ let $\widetilde{A B}: P \rightarrow P ; x \mapsto y$ with $\{y\}=\left[[x]_{2} \cap B\right]_{1} \cap\left[[x]_{1} \cap A\right]_{2}$ and $\widetilde{C}:=\widetilde{C C}$ (we also denote $[X]_{i}:=\bigcup\left\{[x]_{i} \mid x \in X\right\}$ for $i \in\{1,2\}$ and $X \subset P$ ).

Then $\widetilde{C}$ is an involutory antiautomorphism of $(P, \square)$ mapping each chain onto a chain and if $D \in \mathfrak{C}$ is a further chain then the product $\widetilde{C} \circ \widetilde{D}$ is 
an automorphism of $(P, \square)$. Two distinct chains $A, B$ are called orthogonal, denoted by $A \perp B$ if $\widetilde{A}(B)=B$. We have " $A \perp B \Rightarrow B \perp A \Rightarrow \widetilde{A} \circ \widetilde{B}=$ $\widetilde{B} \circ \widetilde{A}$ ". If $\mathfrak{K} \subseteq \mathfrak{C}$ then $\left(P, \mathfrak{G}_{1}, \mathfrak{G}_{2}, \mathfrak{K}\right)$ is called chain structure and the set $\mathfrak{K}^{s}:=\{C \in \mathfrak{C} \mid \widetilde{C}(\mathfrak{K})=\mathfrak{K}\}$ the symmetric stabilizer of $\mathfrak{K}$. A chain structure $\left(P, \mathfrak{G}_{1}, \mathfrak{G}_{2}, \mathfrak{K}\right)$ is called symmetric chain structure if $\forall K \in \mathfrak{K}: \widetilde{K}(\mathfrak{K})=\mathfrak{K}$, i.e. if $\mathfrak{K} \subseteq \mathfrak{K}^{s}$ and symmetric closed if $\mathfrak{K}=\mathfrak{K}^{s}$, see [1-7].

A chain structure $\left(P, \mathfrak{G}_{1}, \mathfrak{G}_{2}, \mathfrak{K}\right)$ is called 2-structure if $\forall(a, b) \in P^{(2)}$ : $\exists_{1} K \in \mathfrak{K}: a, b \in K$ - we set $\overline{a, b}:=K$-, or in other words, if $\left(P, \mathfrak{G}_{1} \cup \mathfrak{G}_{2} \cup \mathfrak{K}\right)$ is an incidence space, i.e. for any two distinct points $a, b \in P$ there is exactly one block $B \in \mathfrak{G}_{1} \cup \mathfrak{G}_{2} \cup \mathfrak{K}$ such that $a, b \in B$. If $(a, b) \in P^{(2)}$ then also $(a \square b, b \square a) \in P^{(2)}$ and we can form the chains $\overline{a, b}$ and $M(a, b):=\overline{a \square b, b \square a}$ called the diagonals of the rectangle $\square(a, b):=(a, a \square b, b, b \square a)$. For $M:=$ $M(a, b)$ we have $\widetilde{M}(a)=b$ and therefore we call $M(a, b)$ the midline of $a$ and $b$.

For a chain structure $\left(P, \mathfrak{G}_{1}, \mathfrak{G}_{2}, \mathfrak{K}\right)$ let $\mathfrak{K}^{2 \perp}$ denote the set of all pairs of orthogonal chains of $\mathfrak{K}, \mathfrak{K}_{0}^{2 \perp}:=\left\{(A, B) \in \mathfrak{K}^{2 \perp} \mid A \cap B=\emptyset\right\}, \mathfrak{K}_{1}^{2 \perp}:=\{(A, B) \in$ $\left.\mathfrak{K}^{2 \perp}|| A \cap B \mid=1\right\}$, and if $p \in P$ and $K \in \mathfrak{K}$ let $(p \perp K):=\{X \in \mathfrak{K} \mid p \in$ $X \wedge X \perp K\}$.

In this paper we consider symmetric 2-structures $\left(P, \mathfrak{G}_{1}, \mathfrak{G}_{2}, \mathfrak{K}\right)$, i.e. the chain structure $\left(P, \mathfrak{G}_{1}, \mathfrak{G}_{2}, \mathfrak{K}\right)$ is at the same time symmetric and a 2 -structure. Let $\Gamma^{+}(\mathfrak{K}):=\operatorname{Aut}(P, \square, \mathfrak{K})$. Then we can state:

Theorem 1.1. 1. The set $\mathfrak{K}^{+}:=\left\{\widetilde{A} \circ \widetilde{B} \mid(A, B) \in \mathfrak{K}^{2 \perp}\right\}$ consists of involutory automorphisms of $\Gamma^{+}(\mathfrak{K})$, is invariant, i.e. $\forall \alpha \in \mathfrak{K}^{+}: \alpha \circ \mathfrak{K}^{+} \circ \alpha=\mathfrak{K}^{+}$, and splits into the two subsets $\mathfrak{K}_{1}^{+}:=\left\{\gamma \in \mathfrak{K}^{+} \mid\right.$Fix $\left.\gamma \neq \emptyset\right\}=\{\widetilde{A} \circ \widetilde{B} \mid$ $\left.(A, B) \in \mathfrak{K}_{1}^{2 \perp}\right\}$ and $\mathfrak{K}_{0}^{+}:=\left\{\gamma \in \mathfrak{K}^{+} \mid\right.$Fix $\left.\gamma=\emptyset\right\}=\left\{\widetilde{A} \circ \widetilde{B} \mid(A, B) \in \mathfrak{K}_{0}^{\perp \perp}\right\}$. Each $\gamma \in \mathfrak{K}_{1}^{+}$is a reflection in a point, i.e. $\gamma$ is involutory, Fix $\gamma$ consists of a single point $c \in P$ and $\gamma(B)=B$ for $B \in\left(\mathfrak{G}_{1} \cup \mathfrak{G}_{2} \cup \mathfrak{K}\right)$ with $c \in B$ [cf. Theorem 3.1.(3)].

2. $\forall(a, b) \in P^{(2)}$ there is a $\gamma \in \mathfrak{K}^{+}$with $\gamma(a)=b$ and the group $\left\langle\mathfrak{K}^{+}\right\rangle$generated by the set $\mathfrak{K}^{+}$acts transitively on $P$ (cf. Theorem 3.10).

3. $\forall p \in P, \forall K \in \mathfrak{K}$ with $p \notin K:|(p \perp K)|=1$ [cf. Theorem 3.1.(5)].

Considering pairs $(p, K) \in P \times \mathfrak{K}$ with $p \in K$ we will show (cf. Theorem 3.11.):

Theorem 1.2. The symmetric 2-structures split into the three classes:

(I) There is a pair $(p, K) \in P \times \mathfrak{K}$ with $p \in K$ and $|(p \perp K)|>1$. Then $\mathfrak{K}_{1}^{+} \neq \emptyset,|(x \perp X)|>1$ to each $x \in P, X \in \mathfrak{K}$ with $x \in X$ and there are several reflections in the point $x$. (If $A \in(x \perp X)$ then $\widetilde{A} \circ \widetilde{X}$ is a reflection in $x$ ) (cf. Theorem 3.12.). For symmetric 2-structures $\left(P, \mathfrak{G}_{1}, \mathfrak{G}_{2}, \mathfrak{K}\right)$ of class $(\mathbf{I})$ we have the bifurcation:

(Ia) $\mathfrak{K}_{1}^{+} \neq \mathfrak{K}^{+}$.

(Ib) $\mathfrak{K}_{1}^{+}=\mathfrak{K}^{+}$. 
(II) There is a pair $(p, K) \in P \times \mathfrak{K}$ with $p \in K:(p \perp K)=\emptyset$. Then for each pair $(x, X) \in P \times \mathfrak{K}$ with $x \in X:(x \perp X)=\emptyset$ and each element of $\mathfrak{K}^{+}$is fixed point free hence $\mathfrak{K}_{1}^{+}=\emptyset$ (cf. Theorem 3.13.).

(III) There is a pair $(p, K) \in P \times \mathfrak{K}$ with $p \in K$ and $|(p \perp K)|=1$. Then for each pair $(x, X) \in P \times \mathfrak{K}$ with $x \in X:|(x \perp X)|=1$ and in $\mathfrak{K}^{+}$ there is exactly one reflection in the point $x$ which we denote by $\widetilde{x}$ and so $\mathfrak{K}_{1}^{+} \neq \emptyset$ (Theorem 3.14.).

For symmetric 2-structures $\left(P, \mathfrak{G}_{1}, \mathfrak{G}_{2}, \mathfrak{K}\right)$ of class (III) we set $\widetilde{P}:=$ (IIIa) $\widetilde{P} \neq \mathfrak{K}^{+}$.

$\{\widetilde{p} \mid p \in P\}$. Then $\widetilde{P}=\mathfrak{K}_{1}^{+}$and we have the biforcation:

(IIIb) $\widetilde{P}=\mathfrak{K}^{+}$.

If $a, b \in P$ are two given points then a point $m$ is called midpoint of $a$ and $b$ if there is $\gamma \in \mathfrak{K}_{1}^{+}$with $\gamma(a)=b$ and Fix $\gamma=\{m\}$.

Theorem 1.3. Let $\left(P, \mathfrak{G}_{1}, \mathfrak{G}_{2}, \mathfrak{K}\right)$ be a symmetric 2-structure and let $a, b \in P$. Then:

1. If $\mathfrak{K}_{1}^{+}=\emptyset$ then $a$ and $b$ do not have a midpoint.

2. If $(a, b) \in P^{(2)}$ then $a$ and $b$ have at most one midpoint and there exists $a$ midpoint if and only if $\overline{a, b} \cap M(a, b) \neq \emptyset$. If $\{m\}:=\overline{a, b} \cap M(a, b) \neq \emptyset$ then $m$ is the midpoint of $a$ and $b$ (cf. Theorem 3.16.).

3. If $\mathfrak{K}_{1}^{+} \neq \emptyset$ and if $a$ and $b$ are parallel points (i.e. $[a]_{1}=[b]_{1}$ or $[a]_{2}=[b]_{2}$ ) then $a$ and $b$ have at least one midpoint. If $\left(P, \mathfrak{G}_{1}, \mathfrak{G}_{2}, \mathfrak{K}\right)$ belongs to the class (III) then a and b have exactly one midpoint [cf. Theorem 3.17.(3)].

4. If $\left(P, \mathfrak{G}_{1}, \mathfrak{G}_{2}, \mathfrak{K}\right)$ belongs to the class (IIIb) or to the class (III) and is symmetrically closed then there is exactly one $\gamma \in \mathfrak{K}^{+}$with $\gamma(a)=b$, i.e. $\left(P, \mathfrak{K}^{+}\right)$ is a regular invariant involutory permutation set [cf. Corollary 3.5.(2) and Theorem 3.18.(2)].

5. If $\left(P, \mathfrak{G}_{1}, \mathfrak{G}_{2}, \mathfrak{K}\right)$ is a symmetric 2-structure such that $\left(P, \mathfrak{G}_{1} \cup \mathfrak{G}_{2} \cup \mathfrak{K}\right)$ is an affine plane then $\left(P, \mathfrak{G}_{1}, \mathfrak{G}_{2}, \mathfrak{K}\right)$ belongs to the class (II) or (IIIb) (cf. Theorem 3.20.).

\section{Notations and Assumptions}

\subsection{Notations Concerning Chain Structures}

In this paper we use the same notations as in [5] hence $\left(P, \mathfrak{G}_{1}, \mathfrak{G}_{2}, \mathfrak{K}\right)$ denotes a chain structure, $\left(P, \mathfrak{G}_{1}, \mathfrak{G}_{2}, \mathfrak{C}\right)$ the corresponding maximal chain structure. A subset $S \subseteq P$ is called subsquare if for all $x, y \in S x \square y \in S$. Let $\Gamma:=\Gamma^{+} \cup \Gamma^{-}$ where $\Gamma^{+}, \Gamma^{-}$denote the sets of all automorphisms and antiautomorphisms of $(P, \square)$ respectively. $\Gamma^{+}$contains for $i=1$ and $i=2$ the following subgroups $\Gamma_{i}:=\left\{\sigma \in \Gamma^{+} \mid \forall x \in P:[\sigma(x)]_{i}=[x]_{i}\right\}$, whose elements are called $i$-maps. We set $\Gamma_{3}:=\Gamma^{+} \backslash\left(\Gamma_{1} \cup \Gamma_{2}\right)$ and if $\alpha \in \Gamma, P_{\alpha}:=\{x \in P \mid(x, \alpha(x))$ $\left.\in P^{(2)}\right\}$. 
Moreover let $\Gamma^{+}(\mathfrak{K}):=\left\{\gamma \in \Gamma^{+} \mid \gamma(\mathfrak{K})=\mathfrak{K}\right\}, \Gamma^{-}(\mathfrak{K}):=\left\{\gamma \in \Gamma^{-} \mid \gamma(\mathfrak{K})=\right.$ $\mathfrak{K}\}, \Gamma(\mathfrak{K}):=\Gamma^{+}(\mathfrak{K}) \cup \Gamma^{-}(\mathfrak{K})$ and $\Gamma_{i}(\mathfrak{K}):=\left\{\gamma \in \Gamma_{i} \mid \gamma(\mathfrak{K})=\mathfrak{K}\right\}$ for $i=1,2,3$. We have:

Theorem 2.1. $\Gamma=\Gamma(\mathfrak{C}), \Gamma^{+}=\Gamma^{+}(\mathfrak{C})$ and $\Gamma^{-}=\Gamma^{-}(\mathfrak{C})$ and

1. $\forall \gamma \in \Gamma^{-} \exists_{1}(A, B) \in \mathfrak{C}^{2}: \gamma=\widetilde{A B}$.

2. $\widetilde{\mathfrak{C}}:=\{\widetilde{A} \mid A \in \mathfrak{C}\}$ is the set of all involutions of $\Gamma^{-}$.

3. $\forall \gamma \in \Gamma^{+}, \forall C \in \mathfrak{C} \exists_{1}(A, B) \in \mathfrak{C}^{2}: \gamma=\widetilde{A B} \circ \widetilde{C}$ and we have:

$\gamma \in \Gamma_{1} \Leftrightarrow B=C$ and then Fix $\gamma=P \square(A \cap C)$,

$\gamma \in \Gamma_{2} \Leftrightarrow A=C$ and then Fix $\gamma=(B \cap C) \square P$.

4. For $\alpha \in \Gamma^{+}: \alpha \in \Gamma_{3} \Leftrightarrow P_{\alpha} \neq \emptyset$.

5. The identity and the set of all involutions of $\Gamma^{+}$consists of all maps of the form $\gamma=\widetilde{A B} \circ \widetilde{C}$ with $\widetilde{C}(A)=A, \widetilde{C}(B)=B \cdot \gamma$ is the identity if and only if $A=B=C \cdot \gamma \in \Gamma_{1}$ is a reflection in the 1-stripe $[A \cap C]_{2}$ if $B=C$ and $\widetilde{C}(A)=A, \quad \gamma \in \Gamma_{2}$ is a reflection in the 2-stripe $[B \cap C]_{1}$ if $A=C$ and $\widetilde{C}(B)=B$ and $\gamma \in \Gamma_{3}$ if $A, B \neq C$ (then Fix $\gamma=[B \cap C]_{1} \cap[A \cap C]_{2}$ is a subsquare).

6. $\widetilde{\mathfrak{C}} \circ \widetilde{\mathfrak{C}} \subseteq \Gamma^{+}$and if $\gamma \in \Gamma^{+}$then

$$
\gamma \in \widetilde{\mathfrak{C}} \circ \widetilde{\mathfrak{C}} \Leftrightarrow \exists C \in \mathfrak{C}: \widetilde{C} \circ \gamma \circ \widetilde{C}=\gamma^{-1} .
$$

If $\mathfrak{K}^{s}:=\{C \in \mathfrak{C} \mid \widetilde{C}(\mathfrak{K})=\mathfrak{K}\}$ denotes the symmetric stabilizer of $\mathfrak{K}$ then $\widetilde{\mathfrak{K}^{s}}:=\left\{\widetilde{A} \mid A \in \mathfrak{K}^{s}\right\}$ is contained in $\Gamma^{-}(\mathfrak{K})$. Let $E \in \mathfrak{K}$ be a fixed chain and for $A, B \in \mathfrak{C}$ let $A \cdot B:=\widetilde{A B}(E)$ - then $(\mathfrak{C}, \cdot)$ is a group. Two distinct chains $A, B \in \mathfrak{C}$ are called orthogonal and denoted by $A \perp B$ if $\widetilde{A}(B)=B$. We have $A \perp B \Rightarrow B \perp A$. Let $\mathfrak{K}^{2 \perp}:=\left\{(A, B) \in \mathfrak{K}^{2} \mid A \perp B\right\}$ and $\mathfrak{K}^{+}:=\left\{\widetilde{A} \circ \widetilde{B} \mid(A, B) \in \mathfrak{K}^{2 \perp}\right\} .\left(P, \mathfrak{G}_{1}, \mathfrak{G}_{2}, \mathfrak{K}\right)$ is called symmetric chain structure if $\mathfrak{K} \subseteq \mathfrak{K}^{s}$.

Theorem 2.2. If $\left(P, \mathfrak{G}_{1}, \mathfrak{G}_{2}, \mathfrak{K}\right)$ is symmetric then $\Gamma(\mathfrak{K})$ contains the group $\langle\widetilde{\mathfrak{K}}\rangle$ generated by the chain reflections $\widetilde{K}$ with $K \in \mathfrak{K}$ and if $A, B \in \mathfrak{K}$ and $\alpha \in \Gamma(\mathfrak{K})$ then:

1. $\widetilde{A} \in \Gamma^{-}(\mathfrak{K})$ and Fix $\widetilde{A}=A$.

2. $\widetilde{A} \circ \widetilde{B} \in \Gamma^{+}(\mathfrak{K})$ and Fix $(\widetilde{A} \circ \widetilde{B})=(A \cap B) \square(A \cap B)$.

3. $\widetilde{A} \circ \widetilde{B}$ is an involution $\Leftrightarrow A \perp B$.

4. $\widetilde{A}(B)=\widetilde{A} \circ \widetilde{B} \circ \widetilde{A}$

5. $\widetilde{\alpha(B)}=\alpha \circ \widetilde{B} \circ \alpha^{-1}$.

\subsection{2-Structures}

In this part we assume that $\left(P, \mathfrak{G}_{1}, \mathfrak{G}_{2}, \mathfrak{K}\right)$ is a 2-structure, i.e. for any $(a, b) \in$ $P^{(2)}$ there is exactly one chain $C \in \mathfrak{K}$ with $a, b \in C$ denoted by $\overline{a, b}:=C$ (called joining line) and since also $(a b, b a) \in P^{(2)}$ we can form the mid-line $M(a, b):=\overline{a b, b a}$. Therefore for any two distinct blocks $A, B \in \mathfrak{B}:=\mathfrak{G}_{1} \cup \mathfrak{G}_{2} \cup \mathfrak{K}$ 
we have $|A \cap B| \leq 1$ and the pair $(P, \mathfrak{B})$ is an incidence space. We also denote $\overline{a, b}:=[a]_{i}$ if $b \in[a]_{i} \backslash\{a\}$ for $i=1,2$.

The set $\mathfrak{K}^{2 \perp}$ of pairs of orthogonal chains splits into the subclasses $\mathfrak{K}_{0}^{2 \perp}:=$ $\left\{(A, B) \in \mathfrak{K}^{2 \perp} \mid A \cap B=\emptyset\right\}$ and $\mathfrak{K}_{1}^{2 \perp}:=\left\{(A, B) \in \mathfrak{K}^{2 \perp}|| A \cap B \mid=1\right\}$ and moreover also the set $P^{(2)}$ of pairs of joinable points splits into $P_{0}^{(2)}:=\{(a, b) \in$ $\left.P^{(2)} \mid \overline{a, b} \cap M(a, b)=\emptyset\right\}$ and $P_{1}^{(2)}:=\left\{(a, b) \in P^{(2)}|| \overline{a, b} \cap M(a, b) \mid=1\right\}$. Also $\mathfrak{K}^{+}$falls into the two subsets $\mathfrak{K}_{1}^{+}:=\left\{\widetilde{A} \circ \widetilde{B} \mid(A, B) \in \mathfrak{K}_{1}^{2 \perp}\right\}$ and $\mathfrak{K}_{0}^{+}:=$ $\left\{\widetilde{A} \circ \widetilde{B} \mid(A, B) \in \mathfrak{K}_{0}^{\perp \perp}\right\}$.

Each pair $(a, b) \in P^{(2)}$ determines a rectangle $\square(a, b):=(a, a b, b, b a)$ with the diagonals $\overline{a, b}$ and $M(a, b)$ and the map $\widetilde{a, b}:=\widetilde{M(a, b)} \circ \widetilde{\widetilde{a, b}}$ of $\Gamma^{+}$ interchanging the points $a$ and $b$ and also $a b$ and $b a$. We have $\mid$ Fix $\widetilde{a, b} \mid=0$ if $(a, b) \in P_{0}^{(2)}$ and $\mid$ Fix $\widetilde{a, b} \mid=1$ if $(a, b) \in P_{1}^{(2)}$. If $(a, b) \in P_{1}^{(2)}$ and $\{d\}=$ $\overline{a, b} \cap M(a, b)$ then $d$ is called the midpoint of $(a, b)$ and of the rectangle $\square(a, b)$ and we have $F i x \widetilde{a, b}=\{d\}$.

Definition 2.3. A collineation $\alpha \in \operatorname{Aut}(P, \mathfrak{B})$ is called point reflection if $\alpha$ is involutory, has exactly one fixed point $p$ and if for $B \in \mathfrak{B}$ with $p \in B$, $\alpha(B)=B$.

It follows that each point reflection is an element of $\Gamma^{+}(\mathfrak{K})$.

Theorem 2.4. Let $\alpha \in \Gamma(\mathfrak{K})$ be a reflection in a point $o, a \in P \backslash\{o\}$ and $X \in \mathfrak{B}$. Then:

1. $\alpha$ is completely determined by the points $o, a$ and $a^{\prime}:=\alpha(a)$,

2. $o \in X \Leftrightarrow \alpha(X)=X$.

Proof. (1) There is an $i \in\{1,2\}$ such that $a \notin[o]_{i}$ for instance $a \notin[o]_{1}$. Then for any $x \in[a]_{1}$ and $x^{\prime}:=\alpha(x)$ we have $\left\{x^{\prime}\right\}=\overline{o, x} \cap\left[a^{\prime}\right]_{1}$. Now let $p \in P \backslash[o]_{2}$, let $\left\{p_{2}\right\}:=[p]_{2} \cap[a]_{1}$ then $\{\alpha(p)\}=\overline{o, p} \cap\left[p_{2}^{\prime}\right]_{2}$ if $p \notin[o]_{1}$ and $\{\alpha(p)\}=[o]_{1} \cap\left[p_{2}^{\prime}\right]_{2}$ if $p \in[o]_{1}$. If $p \in[o]_{2}$ we choose $q \in[p]_{1} \backslash\{p\}$ then $\{\alpha(p)\}=[o]_{2} \cap[\alpha(q)]_{1}$.

(2) " $\Leftarrow$ " Conversely, suppose that $o \notin X$. Then $X \cap[o]_{i} \neq \emptyset$ for $i=1$ or $i=2$. We get a contradiction with Fix $\alpha=\{o\}$.

\section{Symmetric 2-Structures}

In this section let $\left(P, \mathfrak{G}_{1}, \mathfrak{G}_{2}, \mathfrak{K}\right)$ be a symmetric 2 -structure. Then by Theorem 2.2 ., the group generated by the line reflections $\widetilde{L}$ with $L \in \mathfrak{K}$ is contained in the group $\Gamma(\mathfrak{K})$.

\subsection{Involutions}

We have:

Theorem 3.1. Let $(a, b) \in P^{(2)}, C:=\overline{a, b}$ and $D:=M(a, b)$ then:

1. $C$ and $D$ are the diagonals of the rectangle $\square(a, b)$ and we have $C \perp D$. 
2. $\widetilde{a, b}$ is an involution of $\Gamma^{+}(\mathfrak{K})$ interchanging the points $a$ and $b$ as well as $a b$ and $b a$ and with Fix $\widetilde{a, b}=C \cap D$.

3. If $m \in C \cap D \neq \emptyset$ then $\widetilde{a, b}$ is a point reflection in $m$.

4. $\mathfrak{K}^{+}=\left\{\widetilde{a, b} \mid(a, b) \in P^{(2)}\right\}, \mathfrak{K}_{0}^{+}=\left\{\widetilde{a, b} \mid(a, b) \in P_{0}^{(2)}\right\}, \mathfrak{K}_{1}^{+}=\{\widetilde{a, b} \mid(a, b) \in$ $\left.P_{1}^{(2)}\right\}$.

5. For $K \in \mathfrak{K}$ and $p \in P \backslash K$ we have $(p \perp K)=\{\overline{p, \widetilde{K}(p)}\}$.

Proof. (1) $\widetilde{C}$ interchanges the two distinct points $a b$ and $b a$ and since $\left(P, \mathfrak{G}_{1}\right.$, $\left.\mathfrak{G}_{2}, \mathfrak{K}\right)$ is symmetric $\widetilde{C}$ is contained in $\Gamma^{-}(\mathfrak{K})$. Therefore $\widetilde{C}(D)$ is a chain of $\mathfrak{K}$ containing $a b$ and $b a$ and since $\left(P, \mathfrak{G}_{1}, \mathfrak{G}_{2}, \mathfrak{K}\right)$ is a 2-structure we obtain $\widetilde{C}(D)=D$ hence $C \perp D$.

(2) By (1) and Theorem 2.2.(4), $\widetilde{D}=\widetilde{\widetilde{C}(D)}=\widetilde{C} \circ \widetilde{D} \circ \widetilde{C}$ hence $\widetilde{a, b}:=\widetilde{C} \circ \widetilde{D}$ is an involution interchanging the points $a, b$ and the points $a b, b a$. By Theorem 2.1.(2), Fixa, $\widetilde{a}=C \cap D$.

(3) We have only to show: if $B \in \mathfrak{B}$ with $m \in B$ then $\widetilde{a, b}(B)=B$. Let $p \in B \backslash\{m\}$ then by $(2), p$ and $p^{\prime}:=\widetilde{a, b}(p)$ are two distinct points and so since $\left(P, \mathfrak{G}_{1}, \mathfrak{G}_{2}, \mathfrak{K}\right)$ is a 2 -structure, there is exactly one block $A \in \mathfrak{B}$ with $p, p^{\prime} \in A$. Since $\widetilde{a, b}$ is an involutory collineation interchanging $p$ and $p^{\prime}, A$ is a fixed block of $\widetilde{a, b}$. Since $\widetilde{a, b} \in \Gamma^{+}$and $m \in$ Fix $\widetilde{a, b}$ we have $\widetilde{a, b}\left([m]_{i}\right)=[m]_{i}$ (for $i=1$ and $i=2$ ). Therefore at least one of these generators is distinct of $A$, for instance $A \neq[m]_{1}$. Then $A \cap[m]_{1} \subset$ Fix $\widetilde{a, b}$ hence by (2) this is $\{m\}$ and so $m \in A$ showing $A=B$.

(4) Follows from (1). (5) Analogously to (1).

From Theorem 2.2, we obtain:

Theorem 3.2. Let $\sigma \in \Gamma(\mathfrak{K})$ and $(a, b) \in P^{(2)}$ then:

1. $(\sigma(a), \sigma(b)) \in P^{(2)}$

2. $\sigma(\overline{a, b})=\overline{\sigma(a), \sigma(b)}$ and $\overline{\sigma(a), \sigma(b)}=\sigma \circ \widetilde{\widetilde{a, b}} \circ \sigma^{-1}$.

3. $\sigma(M(a, b))=M(\sigma(a), \sigma(b))$.

4. $(\sigma(a), \sigma(b)) \in P_{1}^{(2)} \Leftrightarrow(a, b) \in P_{1}^{(2)}$.

5. $\sigma \widetilde{(a), \sigma(b)}=\sigma \circ \widetilde{a, b} \circ \sigma^{-1}$ and Fix $\widetilde{\sigma(a), \sigma}(b)=\sigma($ Fix $\widetilde{a, b})$.

6. $\mathfrak{K}^{+}, \mathfrak{K}_{1}^{+}$and $\mathfrak{K}_{0}^{+}$are invariant sets of involutions and even the normalizers of each of these sets contain $\Gamma(\mathfrak{K})$.

Theorem 3.3. Let $(a, b) \in P^{(2)}$ and $K \in \mathfrak{K}$ with $\widetilde{a, b}(K)=K$ then there is exactly one $L \in \mathfrak{K}^{s}$ with $\widetilde{a, b}=\widetilde{K} \circ \widetilde{L}$. If $(a, b) \in P_{1}^{(2)}$ or if $\left(P, \mathfrak{G}_{1}, \mathfrak{G}_{2}, \mathfrak{K}\right)$ is symmetrically closed then we have additionally $L \in \mathfrak{K}$.

Proof. $\widetilde{a, b}(K)=K$ implies $\widetilde{K}=\widetilde{\widetilde{a b}(K)}=\widetilde{a b} \circ \widetilde{K} \circ \widetilde{a b}$ Therefore $\alpha:=\widetilde{K} \circ \widetilde{a b}$ is an involution of $\Gamma^{-}(\mathfrak{K})$, i.e. there is an $L \in \mathfrak{K}^{s}$ with $\alpha=\widetilde{L}$ and so $\widetilde{a, b}=\widetilde{K} \circ \widetilde{L}$. 
Let $(a, b) \in P_{1}^{(2)}$. Then, by Theorem 3.1.(3), $\widetilde{a, b}$ is a reflection in a point $m$ and $m \in K$. Let $p \in K \backslash\{m\}, q:=\widetilde{a, b}(p)$ and $L^{\prime}:=\overline{m, p q}$. Then $\widetilde{a, b}\left(L^{\prime}\right)=L^{\prime}$ and $\widetilde{a, b}(p q)=q p$, hence $q p \in L^{\prime}$. By Theorem 3.1.(1),(3) we have $L^{\prime} \perp K$ and $\widetilde{L^{\prime}} \circ \widetilde{K}=\widetilde{p, q}$ is a point reflection. By Theorem 2.4.(1) $\widetilde{L^{\prime}} \circ \widetilde{K}=\widetilde{L} \circ \widetilde{K}$ hence $L=L^{\prime} \in \mathfrak{K}$.

In the above proof we can replace " $\widetilde{a, b} \in \mathfrak{K}_{1}^{+}$" by " $\alpha$ is a point reflection" and we get:

Theorem 3.4. Let $\alpha$ be a point reflection, $K \in \mathfrak{K}$ and $\alpha(K)=K$. Then there is exactly one $L \in \mathfrak{K}$ with $\alpha=\widetilde{K} \circ \widetilde{L}$.

Corollary 3.5. $\quad$ 1. $\mathfrak{K}_{1}^{+}$coincides with the set of point reflections.

2. $\forall(a, b) \in P_{1}^{(2)}$ there is exactly one $\gamma \in \mathfrak{K}_{1}^{+}$with $\gamma(a)=b$.

3. Let $\alpha$ be a reflection in a point $p$ and $K \in \mathfrak{K}$ then $p \in K \Leftrightarrow \widetilde{K} \circ \alpha$ is involutory.

4. Let $p \in P$ and $K, L \in \mathfrak{K}$ with $p \in K, L$ then $|(p \perp K)|=|(p \perp L)|$ is the cardinality of the reflections in the point $p$.

Proof. (1) Follows from Theorem 3.1.(3) and Theorem 3.4.

(2) Let $X=\overline{a, b}, Y=M(a, b)$ and $\{p\}=X \cap Y=$ Fixa, $\widetilde{a, b}$. Assume $\gamma^{\prime}(a)=$ $b$ for any $\gamma^{\prime}=\widetilde{C} \circ \widetilde{D} \in \mathfrak{K}_{1}^{+}$and $\left\{p^{\prime}\right\}=F i x \gamma^{\prime}$. We have $\gamma^{\prime}(X)=X$, $\gamma^{\prime}(Y)=Y$, hence $p^{\prime} \in X, Y$ by Theorem 2.4.(2). Thus $p=p^{\prime}$ and $\gamma^{\prime}=\gamma$ by Theorem 2.4.(1).

(3) " $\Rightarrow$ " Since $\alpha$ is a reflection in a point $p$ and $p \in K$ we have $\alpha(K)=$ $K$ and so by Theorem 3.4., there is a $L \in \mathfrak{K}$ with $\alpha=\widetilde{K} \circ \widetilde{L}$ hence $\widetilde{K} \circ \alpha=\widetilde{K} \circ \widetilde{K} \circ \widetilde{L}=\widetilde{L}$ is an involution. " $\Leftarrow$ " By Theorem 2.2.(5), $\widetilde{\alpha(K)}=\alpha \circ \widetilde{K} \circ \alpha=\widetilde{K}$. Hence $\alpha(K)=K$ and $p \in K$ by Theorem 2.4.(2).

(4) Let $A \in(p \perp K)$ then $\alpha:=\widetilde{K} \circ \widetilde{A}$ is a reflection in the point $p$ hence $\alpha(L)=L$ and by Theorem 3.4, there is exactly one $B \in \mathfrak{K}$ with $\alpha:=\widetilde{L} \circ \widetilde{B}$ and by (3), $p \in B$, i.e. $B \in(p \perp L)$. This shows that the cardinalities of $(p \perp K)$ and $(p \perp L)$ are equal.

Now we consider involutory elements $\alpha$ of $\Gamma(\mathfrak{K})$ and ask if they are contained in $\langle\widetilde{\mathfrak{K}}\rangle$. For each point $x \in P_{\alpha}$ we set: $X:=\overline{x, \alpha(x)}, Y:=\overline{x \square \alpha(x), \alpha(x) \square x}$ and we have: $\alpha(x) \in P_{\alpha}, \alpha(X)=X, \alpha(Y)=Y$ and $\square(x, \alpha(x))$ is a rectangle with the diagonals $X$ and $Y$. Moreover $\widetilde{Y}(x)=\alpha(x)$ and $\widetilde{X}(x \square \alpha(x))=\alpha(x) \square x$ and $X \perp Y$.

Theorem 3.6. If $\alpha \in \Gamma^{-}$then: $\exists_{1} A \in \mathfrak{K}^{s}$ such that $\alpha=\widetilde{A}$ and if $x \in P \backslash A$ then $(x, \alpha(x)) \in P^{(2)}$ and $X \cap Y \subseteq A$.

Proof. By Theorem 2.1.(2) there is exactly one chain $A \in \mathfrak{C}$ with $\alpha=\widetilde{A}$ and by $\widetilde{A}(\mathfrak{K})=\alpha(\mathfrak{K})=\mathfrak{K}$ we have $A \in \mathfrak{K}^{s}$. Since $\alpha$ fixes each of the points $x \square \alpha(x)$ and $\alpha(x) \square x$ we have $x \square \alpha(x), \alpha(x) \square x \in(A \cap Y)$. If we assume $X \cap Y \neq \emptyset$, 
hence (since $\left(P, \mathfrak{G}_{1}, \mathfrak{G}_{2}, \mathfrak{K}\right)$ is a 2 -structure) $\{z\}:=X \cap Y$ consists of a single point, then $\{\widetilde{A}(z)\}=\{\alpha(z)\}=\alpha(X \cap Y)=X \cap Y=\{z\}$ hence $z \in A$.

Definition 3.7. Let $S \subset P$ be a subsquare with $\emptyset \neq S \neq P$. An involution $\alpha \in \Gamma(\mathfrak{K})$ is called reflection in the subsquare $S$ if Fix $\alpha=S$ and if $\forall x \in$ $P \backslash S: \overline{x, \alpha(x)} \cap S \neq \emptyset$.

Theorem 3.8. Let $\alpha \in \Gamma_{3}(\mathfrak{K})$ be an involution hence $P_{\alpha} \neq \emptyset$ and $\mathfrak{K}_{\alpha}:=\{X \mid$ $\left.x \in P_{\alpha}\right\}$ then:

1. If $x \in P_{\alpha}$ then $\alpha(x \square \alpha(x))=\alpha(x) \square x$ (i.e. $\alpha$ interchanges also the points $x \square \alpha(x)$ and $\alpha(x) \square x)$.

2. $\forall K \in \mathfrak{K}: \alpha(K)=K \Leftrightarrow K \in \mathfrak{K}_{\alpha}$.

3. $\forall L \in \mathfrak{K}_{\alpha}: \alpha \circ \widetilde{L}$ is an involution hence $\alpha \circ \widetilde{L} \in \widetilde{\mathfrak{C}}$ and $\exists_{1} L^{s} \in \mathfrak{K}^{s}$ such that $\alpha=\widetilde{L} \circ \widetilde{L^{s}}$.

4. If $|F i x \alpha|>1$ then $\mathfrak{K}_{\alpha}=\left\{\overline{x, y} \mid(x, y) \in F i x \alpha^{(2)}\right\}$.

5. If there is a $x \in P_{\alpha}$ such that $(x, \alpha(x)) \in P_{1}^{(2)}$ then Fix $\alpha \supseteq$ Fix $\widetilde{x, \alpha(x)}$ $\neq \emptyset$.

6. Fix $\alpha$ is a subsquare and for each $L \in \mathfrak{K}_{\alpha}: \widetilde{L}($ Fix $\alpha)=$ Fix $\alpha$, i.e. $L \cap F i x \alpha$ is a chain of Fix $\alpha$ if Fix $\alpha \neq \emptyset$.

7. $P_{\alpha}$ is the complement of the cross $[\text { Fix } \alpha]_{1} \cup[\text { Fix } \alpha]_{2}$ hence $P_{\alpha}=P \backslash$ $\left([F i x \alpha]_{1} \cup[F i x \alpha]_{2}\right)$.

8. If Fix $\alpha \neq \emptyset$ then $\alpha$ is a reflection in the subsquare Fix $\alpha$.

9. If Fix $\alpha$ is a point $p \in P$ and $x \in P_{\alpha}$ then $\alpha$ is a reflection in the point $p$ and $\alpha=\widehat{x, \alpha(x)}$.

10. If $\alpha$ is a reflection in a point $p$ then $\mathfrak{K}_{\alpha}=\{K \in \mathfrak{K} \mid \alpha(K)=K\}=\{K \in$ $\mathfrak{K} \mid p \in K\}$.

Proof. (2) Let $K \in \mathfrak{K}$ with $\alpha(K)=K$. If $K \cap P_{\alpha} \neq \emptyset$ then $K \in \mathfrak{K}_{\alpha}$. Since $\alpha \in \Gamma^{+}, K \subseteq$ Fix $\alpha$ would imply $\alpha=i d$.

(3) Since $\alpha$ is an involution and $\alpha(L)=L$ we have by Theorem 2.2.(5), $\alpha \circ \widetilde{L} \circ \alpha \circ \widetilde{L}=\widetilde{\alpha(L)} \circ \widetilde{L}=\widetilde{L} \circ \widetilde{L}=i d$ hence $\alpha \circ \widetilde{L}$ is an involution of $\Gamma^{-}$and by Theorem 2.1.(2) there is an $X^{\prime} \in \mathfrak{C}$ with $\widetilde{X^{\prime}}=\alpha \circ \widetilde{L}$. Now $\alpha, \widetilde{L} \in \Gamma(\mathfrak{K})$ imply $X^{\prime} \in \mathfrak{K}^{s}$.

(4) Clearly, by $(2), \mathfrak{K}_{\alpha} \supseteq\left\{\overline{x, y} \mid(x, y) \in F i x \alpha^{(2)}\right\}$. Now let $A \in \mathfrak{K}_{\alpha}$ hence there is a point $p \in F i x \alpha \backslash A$ by $|F i x \alpha|>1$ and Theorem 2.1.(5). Then we have two distinct points $x, y \in A$ with $\{x\}:=[p]_{1} \cap A$ and $\{y\}:=[p]_{2} \cap A$ $(x, y) \in P^{(2)}$. Moreover $\{\alpha(x)\}=\alpha\left([p]_{1} \cap A\right)=[\alpha(p)]_{1} \cap \alpha(A)=[p]_{1} \cap A=$ $\{x\}$ and in the same way, $\alpha(y)=y$ hence $\left.(x, y) \in F i x \alpha^{(2)}\right\}$ and $A=\overline{x, y}$.

(5) $(x, \alpha(x)) \in P_{1}^{(2)}$ implies Fixx, $\widehat{\alpha(x)}=\overline{x, \alpha(x)} \cap \overline{x \square \alpha(x), \alpha(x) \square x} \neq \emptyset$ hence by (1), Fixx, $\alpha(x) \subseteq$ Fix $\alpha$.

(6) Let $x \in$ Fix $\alpha$ then by $(3), \widetilde{L}(x)=\widetilde{L} \circ \alpha(x)=\alpha \circ \widetilde{L}(x)$, i.e. $\widetilde{L}(x) \in F i x \alpha$.

(8) By (6), Fix $\alpha$ is a non empty subsquare and for each $x \in P_{\alpha}$ we have $\overline{x, \alpha(x)} \cap$ Fix $\alpha \neq \emptyset$. Now let for instance $x \in[\text { Fix } \alpha]_{1} \backslash$ Fix $\alpha$ and 
$\{y\}:=[x]_{1} \cap$ Fix $\alpha$ then $\alpha(y)=y, x \in[y]_{1}$ and so $\alpha(x) \in[\alpha(y)]_{1}=[y]_{1}$ hence $\overline{x, \alpha(x)}=[y]_{1}$, i.e. $\overline{x, \alpha(x)} \cap$ Fix $\alpha \neq \emptyset$.

(9) The first part is a direct consequence of (8) and for the orthogonal fixed lines $X$ and $Y$ we have $X \cap Y=\{p\}$ and so by Theorem 3.1.(3), $\widetilde{x, \alpha(x)}=$ $\widetilde{X} \circ \tilde{Y}$ is a reflection in the point $p$ interchanging, like $\alpha$, the points $x$ and $\alpha(x)$ hence by Theorem 2.4.(1), $\alpha=\widetilde{x, \alpha(x)}$.

(10) Follows from (2), (6) and Definition 2.3.

Theorem 3.9. Let $\alpha \in\left(\Gamma_{1}(\mathfrak{K}) \cup \Gamma_{2}(\mathfrak{K})\right)$ be a involution then:

1. $\forall A \in \mathfrak{K} \exists_{1} A^{\prime} \in \mathfrak{C}$ such that $A^{\prime} \perp A, \widetilde{A A^{\prime}}(\mathfrak{K})=\mathfrak{K}$ and $\alpha=\widetilde{A^{\prime} A} \circ \widetilde{A}$ if $\alpha \in \Gamma_{1}$ (and then $\alpha$ is a reflection in the stripe Fix $\alpha=\left[A \cap A^{\prime}\right]_{1}$ ) and $\alpha=\widetilde{A A^{\prime}} \circ \widetilde{A}$ if $\alpha \in \Gamma_{2}$ (and then $\alpha$ is a reflection in the stripe Fix $\alpha=\left[A \cap A^{\prime}\right]_{2}$ ).

2. If there are $\left(A, A^{\prime}\right) \in \mathfrak{K} \times \mathfrak{C}$ such that $A^{\prime} \perp A$ and $\widetilde{A A^{\prime}}(\mathfrak{K})=\mathfrak{K}$ then $\alpha_{1}:=\widetilde{A^{\prime} A} \circ \widetilde{A}$ is an involution of $\Gamma_{1}(\mathfrak{K}), \quad \alpha_{2}:=\widetilde{A A^{\prime}} \circ \widetilde{A}$ of $\Gamma_{2}(\mathfrak{K})$ and $\alpha_{1} \circ \alpha_{2}=\widetilde{A^{\prime} A} \circ \widetilde{A^{\prime} A}$ is an involution of $\Gamma_{3}(\mathfrak{K})$ more precisely $\alpha_{1} \circ \alpha_{2}$ is a reflection in the subsquare Fix $\left(\alpha_{1} \circ \alpha_{2}\right)=\left[A \cap A^{\prime}\right]_{1} \cap\left[A \cap A^{\prime}\right]_{2}$.

3. If in (2), $\{a\}:=A \cap A^{\prime} \neq \emptyset$ is a point then $\alpha_{1}$ is a reflection in the generator $[a]_{1}$ and $\alpha_{2}$ in the generator $[a]_{2}$ and moreover $\alpha_{1} \circ \alpha_{2}$ is a reflection in the point $a$.

\subsection{Classification}

Theorem 3.10. The group $\left\langle\mathfrak{K}^{+}\right\rangle$(and so also $\langle\widetilde{\mathfrak{K}}\rangle$ ) acts transitively on the set $P$ of points and we have:

1. If in $\langle\widetilde{\mathfrak{K}}\rangle$ there is a reflection in a point o then there is a reflection in each point $p \in P$.

2. For every pair $(p, K) \in P \times \mathfrak{K}$ with $p \in K$ the set $(p \perp K)$ has the same cardinality.

3. If $(A, B) \in \mathfrak{K}_{1}^{2 \perp}$ then $\widetilde{A} \circ \widetilde{B}$ is a reflection in the point $p$ with $\{p\}=A \cap B$.

4. If $\alpha \in \Gamma_{3}(\mathfrak{K})$ is an involution with Fix $\alpha=\{o\}$ and $a \in P_{\alpha}$ then $\alpha=\widetilde{a, \alpha(a)}$ is a reflection in 0 .

Proof. Let $a, b \in P$. If $(a, b) \in P^{(2)}$ then by Theorem 3.1.(2) $\widetilde{a, b}$ is an involution of $\langle\widetilde{\mathfrak{K}}\rangle$ interchanging $a$ and $b$. If for instance $[a]_{1}=[b]_{1}$, let $c \in P \backslash\left([a]_{1} \cup[a]_{2} \cup\right.$ $\left.[b]_{2}\right)$ then $(a, c),(c, b) \in P^{(2)}$ and so $\widetilde{a, c} \circ \widetilde{c, b}$ is a map of $\langle\widetilde{\mathfrak{K}}\rangle$ taking $b$ onto $a$. If $\alpha$ is a reflection in $o$ and $p \in P$ let $\gamma \in\langle\widetilde{\mathfrak{K}}\rangle$ with $\gamma(o)=p$ then $\gamma \circ \alpha \circ \gamma^{-1}$ is a reflection in $p$.

(2) (2) is now a consequence of Corollary 3.5.(4).

(3) is a consequence of Theorem 3.1.(3) and (4) of Theorem 3.8.(9).

According to Theorem 3.10. we can classify symmetric 2-structures $\left(P, \mathfrak{G}_{1}, \mathfrak{G}_{2}, \mathfrak{K}\right)$ by properties of the set $(p \perp K)$ for any fixed pair $(p, K)$ with $p \in K$. 
Theorem 3.11. The symmetric 2-structures split into the three classes:

(I) There is a pair $(p, K) \in P \times \mathfrak{K}$ with $p \in K$ and $|(p \perp K)|>1$.

(II) There is a pair $(p, K) \in P \times \mathfrak{K}$ with $p \in K$ and $(p \perp K)=\emptyset$.

(III) There is a pair $(p, K) \in P \times \mathfrak{K}$ with $p \in K$ and $|(p \perp K)|=1$

Each of the classes (I) and (III) bifurcate into the subclasses (Ia) and (Ib) and into (IIIa) and (IIIb) depending whether $\mathfrak{K}_{1}^{+} \neq \mathfrak{K}^{+}$or $\mathfrak{K}_{1}^{+}=\mathfrak{K}^{+}$.

Theorem 3.12. Let the symmetric 2-structures be of class (I) Then:

1. To each $A \in(p \perp K) \widetilde{A} \circ \widetilde{K}$ is a reflection in the point $p$ and if $A, B \in$ $(p \perp K)$ with $A \neq B$ then $\widetilde{A} \circ \widetilde{K}$ and $\widetilde{B} \circ \widetilde{K}$ are distinct reflections in the point $p$.

2. $|(x \perp X)|>1$ to each $x \in P, X \in \mathfrak{K}$ with $x \in X$.

3. If $\alpha, \beta$ are two different reflections in the point o then $\alpha \circ \beta=\beta \circ \alpha$ and $\alpha \circ \beta$ is a reflection in the point $o$.

4. If $o \in M$ and $A, B \in(o \perp M)$ then $A \perp B$ or $A=B$.

5. For any $o \in P$ the set of reflections in o with identity constitute an elementary abelian group and the groups are isomorphic for any two points.

Proof. (1) Follows from Theorem 3.1.

(2) Follows from Theorem 3.10.(2).

(3) Let $\alpha=\widetilde{A} \circ \widetilde{B}$. We have $o \in A, B$ and $\beta \circ \alpha \circ \beta=\beta \circ \widetilde{A} \circ \widetilde{B} \circ \beta=$ $\beta \circ \widetilde{A} \circ \beta \circ \beta \circ \widetilde{B} \circ \beta=\widetilde{A} \circ \widetilde{B}=\alpha$. Hence $\alpha \circ \beta$ is an involution with fixed point $o$ i.e. a reflection in the point $o$ by Theorem 3.8.(9).

(4) Suppose $A \neq B$. By Theorem 3.1.(2), $\widetilde{A} \circ \widetilde{M}$ and $\widetilde{M} \circ \widetilde{B}$ are reflections in the point $o$. By (3), $\widetilde{A} \circ \widetilde{M} \circ \widetilde{M} \circ \widetilde{B}=\widetilde{A} \circ \widetilde{B}$ is an involution, hence $B \perp C$.

(5) Follows from (3) and Theorem 3.10.(1).

Theorem 3.13. The following conditions are equivalent:

1. The symmetric 2-structure is of class (II).

2. $(x \perp X)=\emptyset$ for each pair $(x, X) \in P \times \mathfrak{K}$ with $x \in X$.

3. $\mathfrak{K}_{1}^{+}=\emptyset$ i.e. each element of $\mathfrak{K}^{+}$is fixed point free.

Theorem 3.14. The following conditions are equivalent:

1. The symmetric 2-structure is of class (III).

2. For each pair $(x, X) \in P \times \mathfrak{K}$ with $x \in X:|(x \perp X)|=1$.

3. For every $x \in P$ there is exactly one reflection in the point $x$.

For symmetric 2-structures of class (III) if $x \in P$ we denote by $\widetilde{x}$ the unique reflection in the point $x$ and we set $\widetilde{P}:=\{\widetilde{p} \mid p \in P\}$. Then $\widetilde{P}=$ $\mathfrak{K}_{1}^{+}$. The symmetric 2 -structures $\left(P, \mathfrak{G}_{1}, \mathfrak{G}_{2}, \mathfrak{K}\right)$ of class (IIIb) are called point symmetric 2-structures.

\subsection{Midpoints}

We recall: 
Definition 3.15. If $a, b \in P$ are two given points then a point $m$ is called midpoint of $a$ and $b$ if there is $\gamma \in \mathfrak{K}_{1}^{+}$with $\gamma(a)=b$ and Fix $\gamma=\{m\}$.

Theorem 3.16. Let $(a, b) \in P^{(2)}$ then:

1. $a$ and $b$ have a midpoint $\Leftrightarrow(a, b) \in P_{1}^{(2)}$.

2. If $(a, b) \in P_{1}^{(2)}$ then $d:=\overline{a, b} \cap M(a, b)$ is the only midpoint of $a$ and $b$.

Theorem 3.17. If $\mathfrak{K}_{1}^{+} \neq \emptyset$ then any two distinct parallel points $a, b \in P$ (i.e. $[a]_{1}=[b]_{1}$ or $\left.[a]_{2}=[b]_{2}\right)$ have at least one midpoint and if $\left(P, \mathfrak{G}_{1}, \mathfrak{G}_{2}, \mathfrak{K}\right)$ is of class (III) then they have exactly one midpoint.

Proof. If $[a]_{1}=[b]_{1}$ let $c \in[a]_{2} \backslash\{a\}$ then by Theorem 3.10.(1) there is a reflection $\gamma$ in the point $c$ and we have $a \neq d:=\gamma(a) \in[a]_{2}$ and so $(a, d \square b) \in$ $P^{(2)}$. Let $D:=\overline{a, d \square b}, m:=\widetilde{D}(c)$ and $\mu:=\widetilde{D} \circ \gamma \circ \widetilde{D}$. Then $\widetilde{D}(d)=b$ and $\mu(m)=\widetilde{D} \circ \gamma \circ \widetilde{D}(\widetilde{D}(c))=\widetilde{D} \circ \gamma(c)=\widetilde{D}(c)=m$ hence $\mu \in \mathfrak{K}_{1}^{+}$and $\mu$ is a reflection in the point $m$. Moreover $\mu(a)=\widetilde{D} \circ \gamma \circ \widetilde{D}(a)=\widetilde{D} \circ \gamma(a)=\widetilde{D}(d)=b$, i.e. $m$ is a midpoint of $a$ and $b$.

This shows that two parallel points have at least one midpoint.

Now let $\left(P, \mathfrak{G}_{1}, \mathfrak{G}_{2}, \mathfrak{K}\right)$ be of class 3 . and let $m_{1}$ and $m_{2}$ be midpoints of $a$ and $b$ and of $a$ and $d$ respectively. Then by Theorem 3.14.(3) to $m_{i}$ corresponds exactly one reflection in the point $m_{i}$ which we denote by $\widetilde{m}_{i}$ hence $\widetilde{m_{1}}(a)=b$ and $\widetilde{m_{2}}(a)=d$. Let $F:=\overline{a, m_{2} m_{1}}$ then $\widetilde{F}(a)=a$ and $\widetilde{F}\left(m_{1}\right)=m_{2}$ hence $\widetilde{m_{2}}=\widetilde{\widetilde{F}\left(m_{1}\right)}=\widetilde{F} \circ \widetilde{m_{1}} \circ \widetilde{F}$ and so $\widetilde{F} \circ \widetilde{m_{1}}=\widetilde{m_{2}} \circ \widetilde{F}$. This implies $\widetilde{F}(b)=\widetilde{F} \circ \widetilde{m_{1}}(a)=\widetilde{m_{2}} \circ \widetilde{F}(a)=\widetilde{m_{2}}(a)=d$ and so $\widetilde{F}(d b)=\widetilde{F}(b) \square \widetilde{F}(d)=d b$ hence $d b \in F$. We have proved:

Let $(b, d) \in P^{(2)}$ and $F:=\overline{b d, d b}$. If $m_{1}$ and $m_{2}$ are midpoints of $b, b d$ and of $b d, d$ respectively then $m_{2} m_{1} \in F$.

Assume $m_{1}^{\prime}$ is a further midpoint of $b, b d$ then $m_{2} m_{1}, m_{2} m_{1}^{\prime} \in F$ and $m_{2} m_{1}, m_{2} m_{1}^{\prime}$ are parallel. Since $F$ is a chain this implies $m_{2} m_{1}=m_{2} m_{1}^{\prime}$ and so $m_{1}=m_{1}^{\prime}$.

\subsection{Point symmetric 2-Structures}

Theorem 3.18. Let $\left(P, \mathfrak{G}_{1}, \mathfrak{G}_{2}, \mathfrak{K}\right)$ be a point symmetric 2-structures, $K \in \mathfrak{K}$ and let $a, b$ be two distinct points. Then:

1. The set $K_{1}^{\perp}:=\{M \in \mathfrak{K}|M \perp K|, K \cap M \mid=1\}$ is a partition of the set of points $P$.

2. There is exactly one $\gamma \in \mathfrak{K}^{+}$with $\gamma(a)=b$, i.e. $\left(P, \mathfrak{K}^{+}\right)$is a regular invariant involutory permutation set.

3. The $\operatorname{map} \phi: \mathfrak{K}^{+} \rightarrow P ; \tilde{p} \mapsto p$ is a bijection.

4. There is exactly one midpoint of $a, b$.

Proof. (1) By definition of class (III), there is exactly one chain orthogonal to $K$ through any point $p \in K$ and, by $\mathfrak{K}^{2 \perp}=\mathfrak{K}_{1}^{2 \perp}$, we have exactly one such a chain through any $p \in P$. 
(2) ,(4) For $(a, b) \in P^{(2)}$ it follows from $P^{(2)}=P_{1}^{(2)}$ and Theorem 3.5.(2). For parallel points it follows from Theorem 3.17 .

(3) Follows from $\mathfrak{K}^{+}=\mathfrak{K}_{1}^{+}=\widetilde{P}$ and Theorem 3.14.(3).

\subsection{Symmetric 2-Structures with Euclid's Parallel Axiom}

In this section we consider symmetric 2-structures $\left(P, \mathfrak{G}_{1}, \mathfrak{G}_{2}, \mathfrak{K}\right)$ where for $\mathfrak{B}:=\left(\mathfrak{G}_{1} \cup \mathfrak{G}_{2} \cup \mathfrak{K}\right),(P, \mathfrak{B})$ is an affine plane. For $K, L \in \mathfrak{B}$ and $p \in P$ we denote $K \| L$ if $K \cap L=\emptyset$ or $K=L$ and by $(p \| K)$ the block of $\mathfrak{B}$ which is parallel to $K$ and passes through $p$.

Lemma 3.19. Let $\left(P, \mathfrak{G}_{1}, \mathfrak{G}_{2}, \mathfrak{K}\right)$ be a symmetric 2-structure of class (III) such that $(P, \mathfrak{B})$ is an affine plane, let $p, q \in P$ and $K, L, M \in \mathfrak{K}$.

1. If $M \perp K, L$ then $K \| L$.

2. If $p \in L, L \| K$ and $\{M\}=(p \perp K)$ then $M \perp L$.

3. $K \| L \Leftrightarrow \exists M: K, L \perp M$.

4. Let $p^{\prime}=\widetilde{K}(p), p \in L, p^{\prime} \in L^{\prime}$ and $L\|K\| L^{\prime}$. Then $L^{\prime}=\widetilde{K}(L)$ hence $\widetilde{K}(p \| K)=\left(p^{\prime} \| K\right)$.

5. If $K \| L$ and $q \in K$ then $L^{\prime}:=\widetilde{K}(L)=\widetilde{q}(L)$.

6. For any pair $(a, b) \in P^{(2)}$ there is exactly one midpoint of $a, b$.

Proof. (1) If $K, L, M$ have a common point then $K=L$ by Theorem 3.14. If there is a point $a$ with $a \in K, L$ and $a \notin M$ then $K=L$ by Theorem 3.1.(5).

(2) Let be $\left\{L^{\prime}\right\}:=(p \perp M)$. By (1), $L^{\prime} \| K$ hence $L^{\prime}=L$ by Euclid axiom.

(3) Follows from (1) and (2).

(4) If $L^{\prime \prime}=\widetilde{K}(L)$ then we obtain $L^{\prime \prime}=L^{\prime}$ by Euclid axiom.

(5) The statement is clear for $K=L$. Therefore let $K \neq L$ (hence $K \cap L=\emptyset$ ) and let $\{M\}:=(q \perp L)$. Then by (2), $M \perp K, L$ and by Theorem 3.14., $\widetilde{q}=\widetilde{K} \circ \widetilde{M}$ and so $\widetilde{q}(L)=\widetilde{K} \circ \widetilde{M}(L)=\widetilde{K}(L)=L^{\prime}$.

(6) Let $c=a b$. By Theorem 3.17., the midpoints $m_{1}$ of $a, c$ and $m_{2}$ of $b, c$ exist and if $L:=\overline{m_{1}, m_{2}}, d:=\widetilde{L}(c), C:=(c \| L)$ and $D:=(d \| L)$ then by $(4), D=\widetilde{L}(C)$ and by $(5), \widetilde{m_{1}}(C)=\widetilde{m_{2}}(C)=D$. Hence $a, b, d \in D$. For the chain $M:=\overline{c, d}$ we have $\widetilde{M}\left(m_{1}\right)=m_{2}, M \perp L$ and so by $(2), M \perp D$ hence $\widetilde{d}:=\widetilde{M} \circ \widetilde{D}$ is a reflection in the point $d$. Finally $\widetilde{d}(a)=\widetilde{M} \circ \widetilde{D}(a)=$ $\widetilde{M}(a)=\widetilde{M} \circ \widetilde{m_{1}}(c)=\widetilde{M} \circ \widetilde{m_{1}} \circ \widetilde{M}(c)=\widetilde{\widetilde{M}\left(m_{1}\right)}=\widetilde{m_{2}}(c)=b$ and so $d$ is a midpoint of $a$ and $b$ which is uniquely determined by Theorem 3.16.(2).

Theorem 3.20. Let $\left(P, \mathfrak{G}_{1}, \mathfrak{G}_{2}, \mathfrak{K}\right)$ be a symmetric 2-structure such that $(P, \mathfrak{B})$ is an affine plane then $\left(P, \mathfrak{G}_{1}, \mathfrak{G}_{2}, \mathfrak{K}\right)$ belongs to the class (II) or (IIIb).

Proof. If $\left(P, \mathfrak{G}_{1}, \mathfrak{G}_{2}, \mathfrak{K}\right)$ is not in the class (II) then by Theorem 3.13., for each $(p, K) \in P \times \mathfrak{K}$ with $p \in K:(p \perp K) \neq \emptyset$. Let $L, L^{\prime} \in(p \perp K), q \in K \backslash\{p\}$ and $M \in(q \perp K)$. Then by Theorem 3.1.(5), $L \cap M=\emptyset$, i.e. $L=(p \| M)=L^{\prime}$. 
Thus by Theorem 3.11., ( $\left.P, \mathfrak{G}_{1}, \mathfrak{G}_{2}, \mathfrak{K}\right)$ belongs to the class (III) and by Lemma 3.19.(6), for any $(a, b) \in P^{(2)}$ there is a midpoint. Hence by Theorem 3.16.(1), $P^{(2)}=P_{1}^{(2)}$ and so by Theorem 3.1.(4), $\mathfrak{K}^{+}=\mathfrak{K}_{1}^{+}$, i.e. $\left(P, \mathfrak{G}_{1}, \mathfrak{G}_{2}, \mathfrak{K}\right)$ belongs to the class (IIIb).

Open Access. This article is distributed under the terms of the Creative Commons Attribution License which permits any use, distribution, and reproduction in any medium, provided the original author(s) and the source are credited.

\section{References}

[1] Alinovi, B., Karzel, H., Tonesi, C.: Halforders and automorphisms of chain structures. J. Geom. 71, 1-18 (2001)

[2] Gabrieli, E., Im, B., Karzel, H.: Webs related to K-loops and reflection structures. Abh. Math. Sem. Univ. Hamburg 69, 89-102 (1999)

[3] Karzel, H.: INZIDENZGRUPPEN (Vorlesungsausarbeitung von I. Pieper und K. Sörensen), Hamburg (1965)

[4] Karzel, H.: Loops related to geometric structures. Quasigr. Relat. Syst. 15, 47-76 (2007)

[5] Karzel, H., Kosiorek, J., Matraś, A.: Properties of auto-and antiautomorphisms of maximal chain structures and their relations to i-perspectivities. Result. Math. 50, 81-92 (2007)

[6] Karzel, H., Kosiorek, J., Matraś, A.: Automorphisms of symmetric and double symmetric chain structures. Result. Math. 55(3-4), 401-416 (2009)

[7] Karzel, H., Pianta, S., Zizioli, E.: From involution sets, graphs and loops to loopnearrings. In: Nearrings and Nearfields, Proceedings of the Conference on Nearrings and Nearfields, Hamburg, July 27-August 3, 2003, pp. 235-252. Springer, Berlin (2005)

Helmut Karzel

Zentrum Mathematik

T.U. München

80290, Munich, Germany

e-mail: karzel@ma.tum.de

Jarosław Kosiorek and Andrzej Matraś

Faculty of Mathematics and Computer Science

University of Warmia and Mazury

Słoneczna 54, 10-710 Olsztyn, Poland

e-mail: kosiorek@matman.uwm.edu.pl;

matras@uwm. edu.pl

Received: October 15, 2010.

Accepted: November 2, 2012. 